\title{
Galanthamine improves myocardial ischemia-reperfusion-induced cardiac dysfunction, endoplasmic reticulum stress-related apoptosis, and myocardial fibrosis by suppressing AMPK/Nrf2 pathway in rats
}

\author{
Xiaolin $\mathrm{Hou}^{1}$, Minhuan $\mathrm{Fu}^{2}$, Biao Cheng ${ }^{2}$, Yu Kang ${ }^{3}$, Dili Xie ${ }^{2}$ \\ ${ }^{1}$ Department of Cardiology, ${ }^{2}$ Department of Geriatric Cardiology, Sichuan Academy of Medical Science \& Sichuan Provincial People's Hospital, \\ Chengdu 610072, China; ${ }^{3}$ Department of Cardiology, West China Medical College, Sichuan University, Chengdu 610065, China \\ Contributions: (I) Conception and design: X Hou, M Fu; (II) Administrative support: X Hou; (III) Provision of study materials or patients: X Hou, M \\ Fu, B Cheng, Y Kang, D Xie; (IV) Collection and assembly of data: All authors; (V) Data analysis and interpretation: X Hou, M Fu; (VI) Manuscript \\ writing: All authors; (VII) Final approval of manuscript: All authors. \\ Correspondence to: Dili Xie. Department of Geriatric Cardiology, Sichuan Provincial People's Hospital, No. 18, Section 3, Renmin South Road, \\ Chengdu 610072, China. Email: ilg7ky21bjebr@sina.com.
}

Background: Myocardial ischemia/reperfusion (I/R) injury is an important cause of myocardial infarction and heart failure after cardiovascular surgery. Galanthamine $(\mathrm{Gal})$ is an important Amaryllidaceae alkaloid with anti-acetylcholinesterase and anti-inflammatory activity. The purpose of this study was to investigate the role of $\mathrm{Gal}$ in myocardial I/R injury.

Methods: In this study, an animal model of myocardial I/R injury was constructed, and the rats were divided into five groups ( $\mathrm{n}=10)$ : the sham, I/R model, I/R + Gal $(1 \mathrm{mg} / \mathrm{kg}), \mathrm{I} / \mathrm{R}+\mathrm{Gal}(3 \mathrm{mg} / \mathrm{kg})$, and $\mathrm{I} / \mathrm{R}+$ Aspirin $(20 \mathrm{mg} / \mathrm{kg})$ groups. The expression of related proteins was detected by Western blotting and Immunohistochemistry, and Histological lesion was detected by HE staining.

Results: Results showed that Gal improves I/R_induced cardiac dysfunction in rats. Moreover, Gal inhibits I/R-induced endoplasmic reticulum stress (ERS)-related apoptosis by suppressing the expression of CHOP, Cleaved caspase 12, and caspase 3, and promoting the expression of CADD34 and BiP in rats. Furthermore, Gal mitigates I/R - induced myocardial fibrosis through restraining the expression of $\alpha$-SMA and Collagen I in rats. Mechanically, Gal promoted the expression of AMPK 1 , Nrf2 and HO-1. However, AMPK inhibitor Compound $\mathrm{C}$ exhibited the opposite effects. Collectively, this finding suggests that Gal improves I/R-induced cardiac dysfunction, ERS-related apoptosis, and myocardial fibrosis by activating AMPK/Nrf2 pathway in myocardial I/R rats.

Conclusions: Given this evidence, Gal may be a potential therapeutic drug for the treatment of I/R injury.

Keywords: Myocardial ischemia/reperfusion injury; galanthamine, endoplasmic reticulum stress; adenosineactivated protein kinase; nuclear factor erythroid-2-related factor 2

Submitted Sep 10, 2019. Accepted for publication Sep 23, 2019.

doi: 10.21037/atm.2019.10.108

View this article at: http://dx.doi.org/10.21037/atm.2019.10.108

\section{Introduction}

Myocardial ischemia does a certain degree of damage to the function, morphology, and metabolism of cardiomyocytes (1).
Restoring blood reperfusion is the most effective way to reduce myocardial ischemia injury. However, the restoration of blood reperfusion will aggravate the damage of cardiomyocyte 
function, structure, and metabolism; i.e., myocardial ischemia/reperfusion (I/R) injury (2). Myocardial I/R injury is a major cause of myocardial infarction and heart failure after cardiovascular surgery (3). With the help of mechanical or drug intervention, the rapid recovery of blood supply through an occlusive coronary artery is the most effective treatment for ischemia (4). However, the mechanism of myocardial I/R injury is very complex (5). Myocardial I/R injury has become a major obstacle in the clinical treatment of ischemic cardiomyopathy (6). Therefore, it is very important to clarify the mechanism of myocardial I/R and to find drugs against myocardial I/R injury for the treatment of ischemic cardiomyopathy.

Galanthamine (Gal) is an important Amaryllidaceae alkaloid with anti-acetylcholinesterase and antiinflammatory activity (7). As an acetylcholinesterase inhibitor, Gal has been approved as a long-acting anticholinesterase drug for the treatment of Alzheimer's disease (AD) (8). The choline-like drugs such as Gal have an effect on the GCI/R injury caused by low cholinergic function (9). Activation of the neuronal or non-neuronal cholinergic pathway in the heart reduces ischemic injury and plays an important role in improving cardiac systolic function and reducing infarct size (10). This study elucidated the role and potential molecular mechanisms of Gal in myocardial I/R.

The endoplasmic reticulum (ER) is a dynamic and stable organelle involved in the synthesis of proteins and lipids and the regulation of $\mathrm{Ca}^{2+}$ concentration (11). Cells exposure to hypoxia or certain chemotherapeutic drugs can cause accumulation of unfolded proteins and changes in calcium homeostasis, leading to endoplasmic reticulum stress (ERS) (12). Under stress conditions, ERS activates adenosine-activated protein kinase (AMPK), a metabolically sensitive protein kinase that regulates cell homeostasis and reprogramming of metabolism. ERS also induces nuclear factor erythroid 2 related factor 2 (Nrf2) nuclear translocation in a PERK-dependent manner without the involvement of subunit eukaryotic translation initiation factor 2 (eIF2a) or ROS accumulation $(13,14)$. Under stress conditions, activation of AMPK and Nrf2 reduces myocardial I/R injury and improves cardiac function $(15,16)$.

This study constructed an animal model of myocardial $\mathrm{I} / \mathrm{R}$ injury and investigated the role of Gal in myocardial $\mathrm{I} / \mathrm{R}$ injury along with the potential mechanism involved. The results found that Gal improves I/R-induced cardiac dysfunction, ERS-related apoptosis, and myocardial fibrosis by suppressing AMPK/Nrf2 pathways in myocardial $\mathrm{I} / \mathrm{R}$ rats. This study indicates that Gal may be a potential therapeutic drug for the treatment of $\mathrm{I} / \mathrm{R}$ injury.

\section{Methods}

\section{Construction of myocardial I/R rat model}

All animal experiments were performed in accordance with the NIH Guide for the Care and Use of Laboratory Animals and were approved by the Medical Ethics Committee of Sichuan Provincial People's Hospital. A total of 50 SpragueDawley (SD) rats (male, weight 220-280 g) were obtained from the Animal Center of Sichuan Provincial People's Hospital and housed in a controlled environment at $25 \pm 3{ }^{\circ} \mathrm{C}$, with a humidity of $60 \%$, in a 12 -h light/dark cycle with free access to water. Rats were grouped into five groups (10 in each group): the sham, I/R model, $\mathrm{I} / \mathrm{R}+\mathrm{Gal}(1 \mathrm{mg} / \mathrm{kg}), \mathrm{I} / \mathrm{R}+\mathrm{Gal}(3 \mathrm{mg} / \mathrm{kg})$, and I/R + Aspirin $(20 \mathrm{mg} / \mathrm{kg})$ group. The rats in the sham group and $\mathrm{I} / \mathrm{R}$ group were slowly injected with normal saline $30 \mathrm{~min}$ before the establishment of the model. The rats in $\mathrm{I} / \mathrm{R}+\mathrm{Gal}$ $(1 \mathrm{mg} / \mathrm{kg})$ and $\mathrm{I} / \mathrm{R}+\mathrm{Gal}(3 \mathrm{mg} / \mathrm{kg})$ group were injected with Gal $(1$ and $3 \mathrm{mg} / \mathrm{kg})$. The rats in $\mathrm{I} / \mathrm{R}+$ Aspirin $(20 \mathrm{mg} / \mathrm{kg})$ group were injected with Aspirin $(20 \mathrm{mg} / \mathrm{kg})$. A myocardial I/R rat model was prepared by a method described by Ahmed et al. (17). Rats were anesthetized by intraperitoneal injection of chloral hydrate $(10 \%)$ and fixed in the supine position. The pericardium of the rat was cut open to expose the heart. A 4-0 suture needle was passed through 2 to $3 \mathrm{~mm}$ below the left atrial appendage, and a $1.5 \mathrm{~mm}$ diameter latex tube was placed under the ligature. Tightening the ligature blocks the blood flow to the coronary arteries to cause myocardial ischemia. After half an hour, the ligature was released and a $2 \mathrm{~h}$ blood perfusion was performed to form a model of ischemia-reperfusion injury. The changes of left ventricular ejection fraction (LVEF), shortening fraction (FS), left ventricular wall thickness (LVWT), left ventricular end-systolic volume (LVESV), and left ventricular end-diastolic volume (LVEDV) were detected by echocardiography.

\section{Western blotting}

Myocardial tissues were lysed in lysis buffer (Beyotime, Shanghai, China) containing protease and phosphatase inhibitor. Proteins $(20 \mu \mathrm{g})$ were separated by $10 \%$ SDSPAGE and transferred to a PVDF membrane. Subsequently, 
the membranes were blocked with $5 \%$ skim milk powder and incubated overnight at $4{ }^{\circ} \mathrm{C}$ with primary antibodies against CHOP (\#2895, 1:1000, Cell Signaling Technology, Beverly, MA, USA), Cleaved caspase 12 (\#2202, 1:1000, CST, Beverly, MA, USA), GADD34 (ab9869, 1:5000, Abcam, Cambridge, UK), Bip (\#3177, 1:1000, CST, Beverly, MA, USA), Actin (ab179467, 1:5000, Abcam, Cambridge, UK). Bcl-2 (\#3498, 1:1000, CST, Beverly, MA, USA), Bax (\#14796, 1:1000, CST, Beverly, MA, USA), $\alpha$-SMA (\#19245, 1:1000, CST, Beverly, MA, USA), Collage I (ab34710, 1:5000, Abcam, Cambridge, UK), AMPKa (ab32047, 1:5000, Abcam, Cambridge, UK), Nrf2 (ab137550, 1:5000, Abcam, Cambridge, UK), and HO-1 (ab13243, 1:5000, Abcam, Cambridge, UK). Then, the membranes were washed with TBST, incubated with secondary antibodies (Abcam, Cambridge, UK) for $1.5 \mathrm{~h}$ at room temperature, and detected using ECL kit (Millipore Corp, Bedford, MA, USA).

\section{Quantitative real-time PCR}

Total RNA was extracted from myocardial tissues using RNAprep pure Tissue Kit (Tiangen, Beijing, China) and reversed transcribed into cDNA using FastKing gDNA Dispelling RT SuperMix according to the manufacturer's instructions. Quantitative real-time PCR was performed using a FastFire qPCR PreMix (SYBR Green) on a 7500 Real-Time PCR System (Applied Biosystems, USA). Primer sequences were as follows: Caspase 3, S, 5'-TCGACTAGCAATGCTGACGC-3'; AS, 5'-CATCGACATCGCTACAATCG-3'.

\section{Immunobistochemistry}

Immunohistochemistry was conducted to detect the expression of caspase 3 in myocardial tissues. Briefly, the slices were deparaffinized with xylene and hydrated with gradient ethanol. Then, the slices were incubated with the primary antibody caspase 3 (\#14214, 1:1000, CST, Beverly, MA, USA), treated with a universal secondary antibody (\#7074, CST, Beverly, MA, USA), and counterstained with hematoxylin. The number of caspase 3-positive cells was counted and expressed as an average number.

\section{Haematoxylin and eosin $(H \& E)$ staining}

Myocardial tissues were fixed in 4\% paraformaldehyde for
$48 \mathrm{~h}$. Then, the tissues were ethanol-dehydrated, paraffinembedded, and sectioned into slices $(5 \mu \mathrm{m})$. Subsequently, the slices were stained with $\mathrm{H} \& \mathrm{E}$ for histological assay.

\section{Statistical analysis}

The data are expressed as mean \pm SEM, and SPSS 17.0 (SPSS, Chicago, IL, USA) was applied to determine statistical significance by unidirectional ANOVA. $\mathrm{P}<0.05$ was considered to be statistically significant.

\section{Results}

\section{Gal improves $I / R$-induced cardiac dysfunction in rats}

The results found that compared with the sham group, the I/R group had LVWT, FS, and LVEF significantly decrease from $0.75 \pm 0.05,27 \pm 4.22$, and $53 \% \pm 6.15 \%$ to $0.61 \pm 0.03,13 \pm 2.61$, and $29 \% \pm 7.08 \%$, respectively, while LVEDV $(\mu \mathrm{L})$ and LVESV $(\mu \mathrm{L})$ were significantly increased from $56 \pm 7.32$ and $31 \pm 4.66$ to $82 \pm 7.50$ and $53 \pm 6.27$, respectively. Interestingly, after Gal treatment, the levels of LVWT, FS, and LVEF were significantly increased in a dose-dependent manner from $0.61 \pm 0.03,13 \pm 2.61$, and $29 \% \pm 7.08 \%$ to $0.71 \pm 0.04$, $22 \pm 4.15$, and $45 \% \pm 6.19 \%$, respectively, while the levels of LVEDV $(\mu \mathrm{L})$ and LVESV $(\mu \mathrm{L})$ were significantly reduced from $82 \pm 7.50$ and $53 \pm 6.27$ to $64 \pm 6.11$ and $41 \pm 4.02$, respectively (Figure 1). A similar effect was observed after treatment with Aspirin. These results indicate that Gal, like Aspirin, improves I/R-induced cardiac dysfunction in rats.

\section{Gal inbibits I/R—induced apoptosis by suppressing endoplasmic reticulum patbway in rats}

We then investigated the roles of Gal in I/R-induced apoptosis. The results showed that, compared with the sham group, I/R treatment significantly promoted the activation of ERS-related pro-apoptotic molecules (CHOP and Cleaved caspase-12), while inhibiting the expression of ERS-associated pro-survival molecules (GADD34 and BiP) (Figure 2A,B). Moreover, RT-qPCR and IHC analysis showed that $\mathrm{I} / \mathrm{R}$ treatment also promoted the expression of caspase 3 in myocardial tissues. Interestingly, Gal treatment dose-dependently inhibited the activation of CHOP and Cleaved caspase-12, while promoting the expression of GADD34 and BiP. At the same time, Gal treatment also promoted the expression of caspase 3 in myocardial tissues in a dose-dependent manner. Similar results were obtained 
A

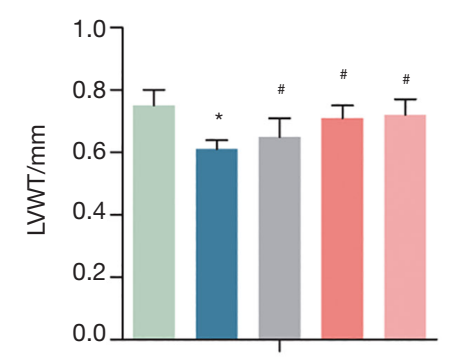

$\mathrm{D}$

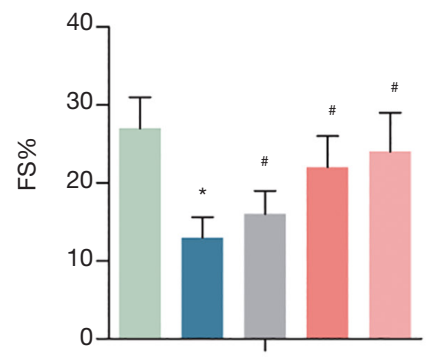

B

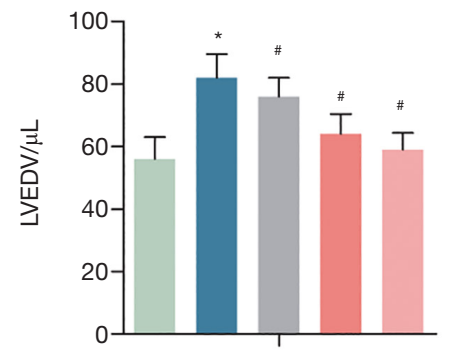

$\mathrm{E}$

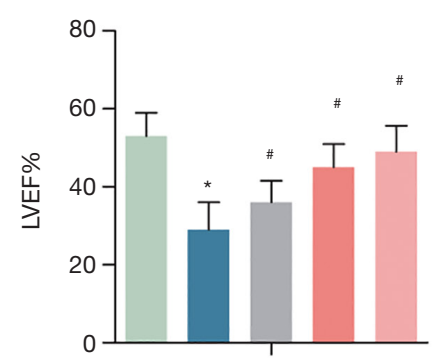

C

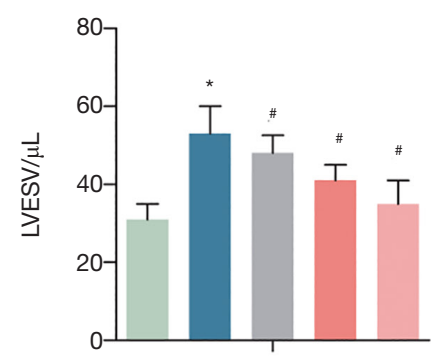

Figure $1 \mathrm{Gal}$ improves I/R-induced cardiac dysfunction in rats. Rats were grouped into five groups (10 in each group): Sham group, I/ R model, $\mathrm{I} / \mathrm{R}+\mathrm{Gal}(1 \mathrm{mg} / \mathrm{kg}), \mathrm{I} / \mathrm{R}+\mathrm{Gal}(3 \mathrm{mg} / \mathrm{kg})$, and I/R + Aspirin (20 mg/kg) group. (A) LVWT; (B) LVEDV; (C) LVESV; (D) FS; (E) LVEF. *, $\mathrm{P}<0.05$ vs. sham group; ${ }^{\#}, \mathrm{P}<0.05$ vs. I/R group.

when treating I/R rats with Aspirin (Figure 2C,D). These results indicate that $\mathrm{Gal}$ inhibits $\mathrm{I} / \mathrm{R}$-induced apoptosis by suppressing endoplasmic reticulum pathway in rats.

\section{Gal mitigates $I / R$-induced myocardial fibrosis in rats}

$\mathrm{H} \& \mathrm{E}$ staining was employed to investigate the effect of $\mathrm{Gal}$ on I/R-induced myocardial fibrosis. The results showed that compared with Sham group, myocardial fibers in the myocardial tissue of the I/R group were arranged in a disorderly fashion and accompanied by nuclear pyknosis. Myocardial tissue was dropsical, and the myocardial space was enlarged. After Gal treatment, the degrees of myocardial fiber disorder and interstitial edema in rat myocardial tissue were improved in a dose-dependent manner compared with the I/R group. Similar effects were observed in the Aspirin group (Figure $3 A$ ). Additionally, western blotting analysis showed that I/R treatment significantly promoted the expression of cardiac fibroblast activation markers $\alpha$-SMA and Collagen I. Interestingly, Gal treatment, like Aspirin, dose-dependently counteracted the effect of I/R on the expression of $\alpha$-SMA and Collagen I (Figure 3B). These results indicate that Gal mitigates I/Rinduced myocardial fibrosis in rats.
Gal improves myocardial I/R injury by activating AMPK/ Nrf2 pathway

To investigate the potential molecular mechanism, western blotting was performed. As shown in Figure 4A, I/R treatment significantly inhibited the expression of AMPK $\alpha 1$ and Nrf pathway-associated proteins (Nrf2 and HO-1) compared to the sham group. However, Gal treatment dose-dependently reversed the inhibitory effect of I/R on AMPK $\alpha 1, \mathrm{Nrf} 2$, and HO-1 expression. These results are similar to those after aspirin treatment of I/R (Figure $4 A$ ). To further verify that the AMPK pathway is involved in the role of Gal, the AMPK pathway inhibitor Compound C (CC) was applied. As shown in Figure 4B, in contrast to $\mathrm{Gal}$, the addition of $\mathrm{CC}$ further inhibited the expression of AMPK $\alpha 1$. Functionally, the addition of CC also aggravates the effects of I/R on cardiac dysfunction, ERS-induced apoptosis, and myocardial fibrosis (Figure $4 C, D, E, F$ ). Collectively, these results demonstrate that Gal improves myocardial I/R injury by regulating AMPK/Nrf2 pathway.

\section{Discussion}

I/R can cause oxidative stress and energy metabolism 
A
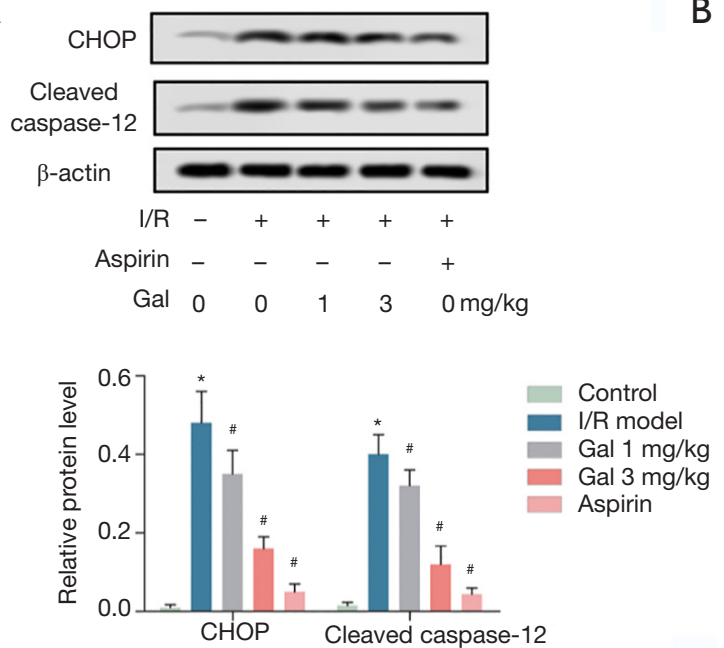

C

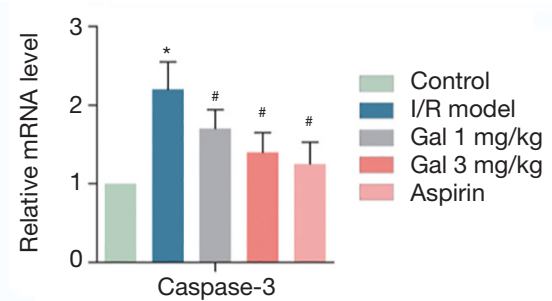

D

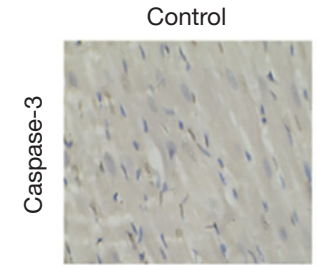

I/R model

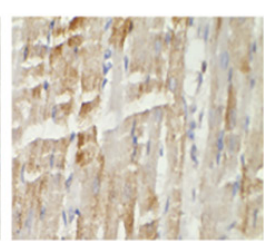

B
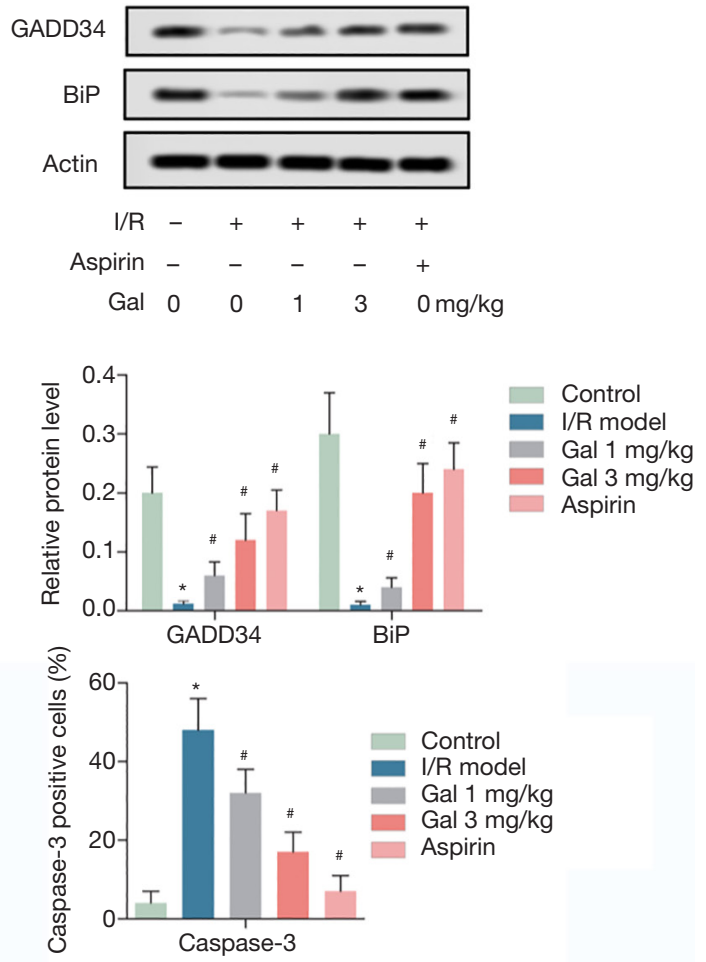

Gal $1 \mathrm{mg} / \mathrm{kg}$

Gal 3 mg/kg

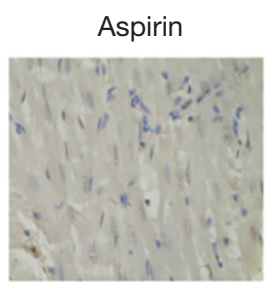

Figure $2 \mathrm{Gal}$ inhibits I/R-induced apoptosis by suppressing endoplasmic reticulum pathway in rats. Rats were grouped into five groups (10 in each group): Sham group, I/R model, I/R + Gal (1 mg/kg), I/R + Gal (3 mg/kg), and I/R + Aspirin (20 mg/kg) group. (A) The protein levels of CHOP and Cleaved caspase 12 were measured by western blotting assay; (B) the protein levels of GADD34 and BiP were measured by western blotting assay; (C) the mRNA level of Caspase 3 was measured by RT-qPCR; (D) the expression of caspase 3 in myocardial tissues was detected by IHC assay. Actin was used as an internal reference. *, $\mathrm{P}<0.05$ vs. sham group; ${ }^{\#}, \mathrm{P}<0.05$ vs. I/R group.

disorder in myocardial tissue, which in turn leads to cardiomyocyte injury, apoptosis, or necrosis $(18,19)$. In addition, it can further induce the activation of ERspecific apoptosis protein Caspase-12 and suppress the expression of CHOP and other proteins by regulating calcium balance to induce endoplasmic reticulum stress $(18,20-22)$. In this study, it was found that after myocardial I/R treatment, LVWT, FS, and LVEF decreased significantly, while LVEDV and LVESV increased significantly. Also, myocardial I/R treatment accelerated the expression of apoptosis-promoting proteins $\mathrm{CHOP}$ and Cleaved caspase-12 in the endoplasmic reticulum, and inhibited the expression of survival-promoting molecules GADD34 and BiP, which promoted apoptosis induced by endoplasmic reticulum stress. In addition, myocardial I/R treatment promoted myocardial fibrosis by promoting the expression of $\alpha$-SMA and Collagen I. These results suggest that myocardial I/R leads to myocardial injury by aggravating cardiac dysfunction, accelerating endoplasmic reticulum stress cell apoptosis, and promoting myocardial fibrosis.

On the one hand, as an acetylcholinesterase inhibitor 
A

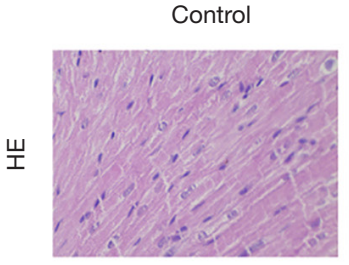

I/R model

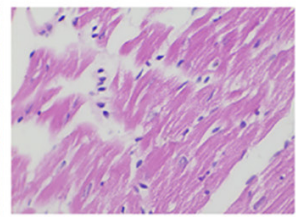

Gal $1 \mathrm{mg} / \mathrm{kg}$

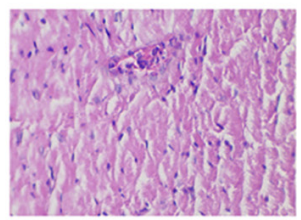

Gal $3 \mathrm{mg} / \mathrm{kg}$

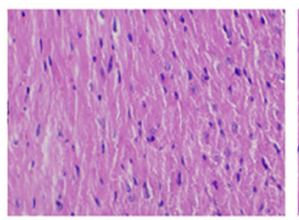

Aspirin

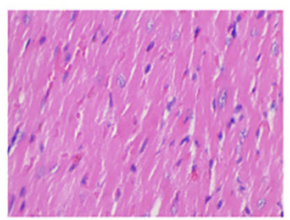

B
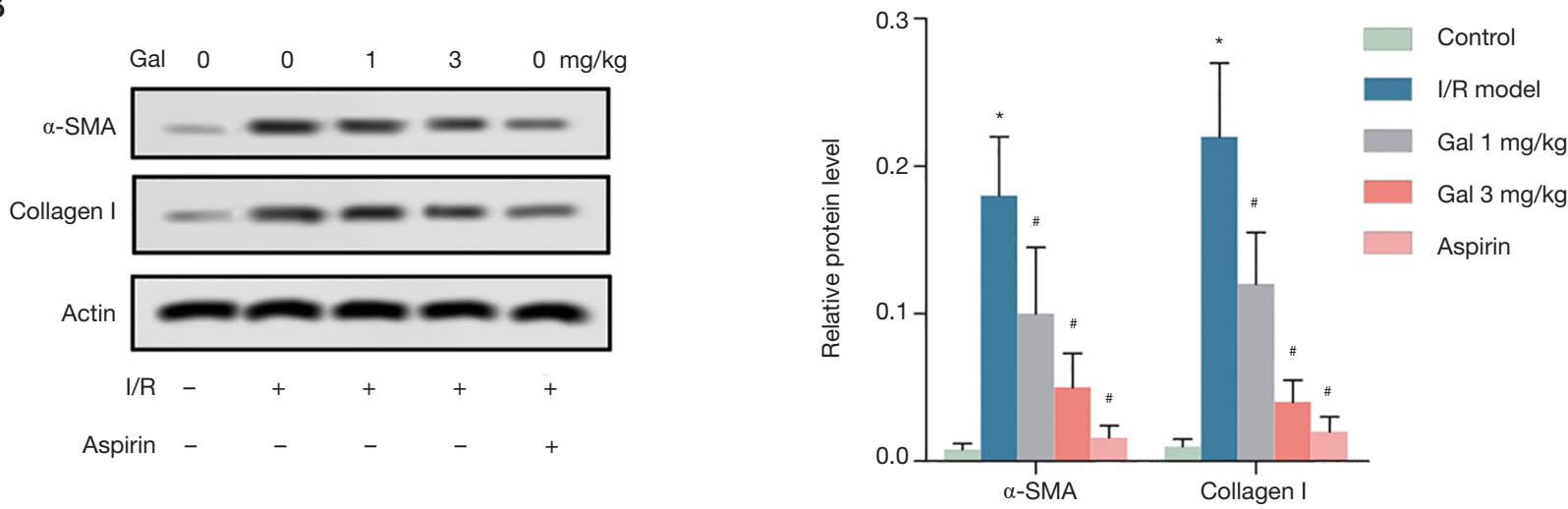

Figure $3 \mathrm{Gal}$ mitigates I/R-induced myocardial fibrosis in rats. Rats were grouped into five groups (10 in each group): Sham group, I/R model, I/R + Gal (1 mg/kg), I/R + Gal (3 mg/kg), and I/R + Aspirin (20 mg/kg) group. (A) Myocardial fibrosis was determined by H\&E staining (magnification: 400x). (B) The protein levels of $\alpha$-SMA and collagen I were measured by western blotting assay. Actin was used as an internal reference. *, $\mathrm{P}<0.05$ vs. sham group;, $\mathrm{P}<0.05$ vs. I/R group.

(AChEI), Gal is widely used in the treatment of Alzheimer's disease and other dementia $(23,24)$. Previous studies have shown that Gal can improve behavioral flexibility disorder and cortical injury caused by traumatic brain injury (25). In addition, Gal has been observed reversing the cognitive impairment of mice after operation, reducing the levels of microglia and inflammatory factors (IL-1 $\beta$, IL-6) in hippocampus, and normalizing early POCD by enhancing cholinergic nerve tension in the hippocampus (26). For one, as a positive allosteric regulator of nicotine acetylcholine receptor (NAChRs), Gal plays an important role in protecting muscarinic and nicotine receptor functions and improving global cerebral ischemia/reperfusion (GCI/R) injury (9). For another, Gal can reduce ischemic injury by protecting muscarinic acetylcholine receptors and activating neuronal or non-neuronal cholinergic pathways in the heart. Furthermore, Gal can inhibit Abeta-induced apoptosis of PC12 cells by improving mitochondrial dysfunction and reducing endoplasmic reticulum stress (27). On this basis, current studies have found that Gal can improve cardiac dysfunction induced by myocardial I/R in a dose-dependent manner, inhibit the expression of endoplasmic reticulum apoptosis-related proteins (CHOP and Cleaved caspase-12) and caspase 3, and promote the expression of GADD34 and $\mathrm{BiP}$ to improve endoplasmic reticulum stress cell apoptosis. Further to this, our study also found that Gal can improve myocardial fibrosis by inhibiting the expression of $\alpha$-SMA and Collagen I. These results suggest that Gal can improve cardiac dysfunction, apoptosis, and myocardial fibrosis caused by myocardial I/R.

Long-term ERS induces ERS-related apoptosis (28). $\mathrm{I} / \mathrm{R}$ can cause myocardial damage by inducing excessive myocardial ERS (29). Therefore, detecting the expression of the endoplasmic reticulum-associated proteins may reflect the effect of I/R on ERS-induced apoptosis. In our study, I/R was found to significantly promote the expression of CHOP and Cleaved caspase 12, while inhibiting the expression of GADD34 and BiP, indicating that $\mathrm{I} / \mathrm{R}$ induces ERS-related apoptosis. MAPK, PI3K/AKT, PERK, JAK/ STAT3, AMPK, and Nrf2 signaling pathways are involved in I/R-induced ERS (30-35). Among them, the activation of AMPK and Nrf2 signaling pathways has a significant 
A

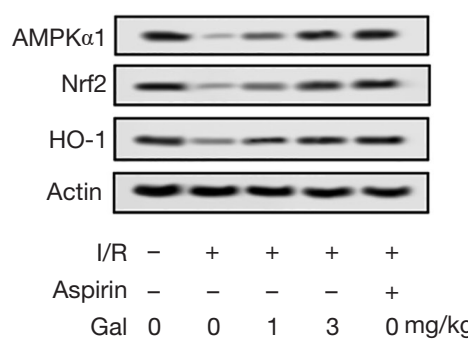

B

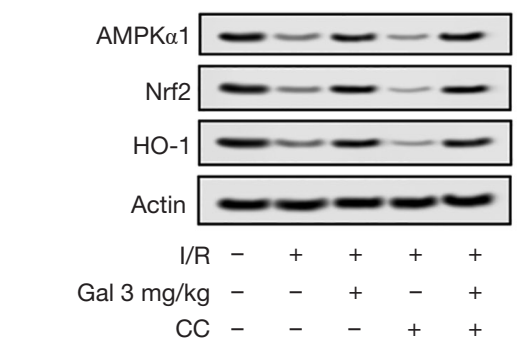

C

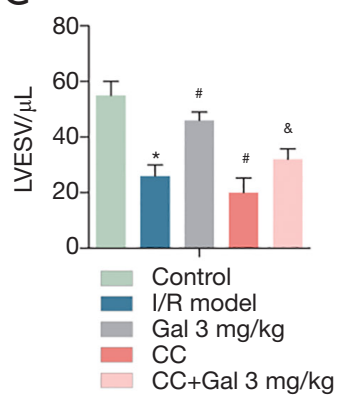

E

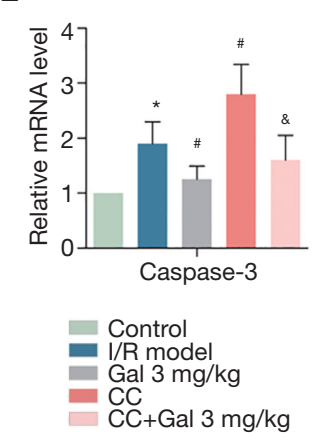

$B$

$\mathrm{F}$

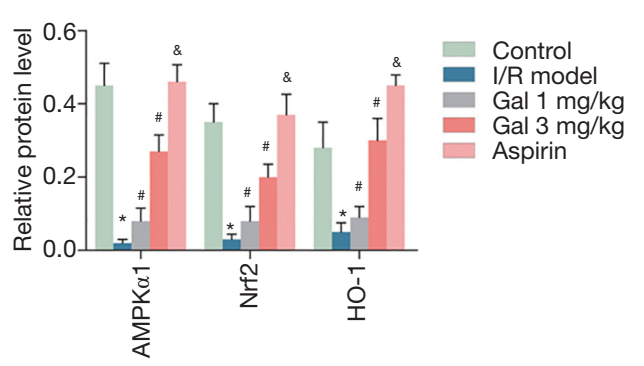

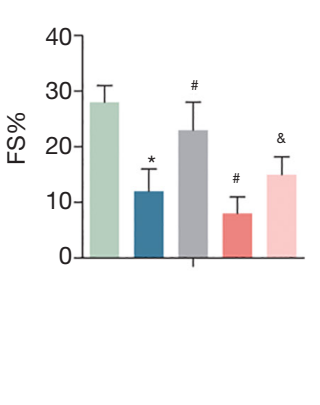

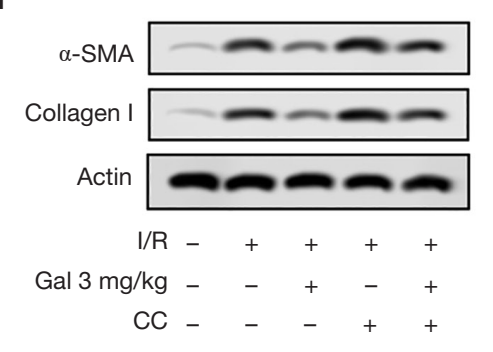

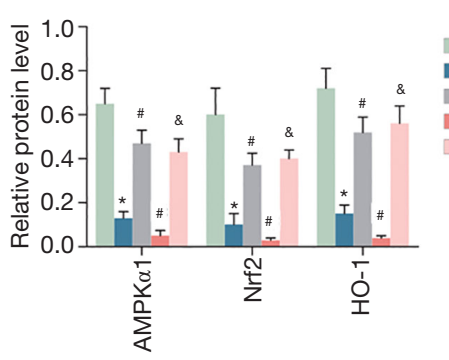

D

$$
\begin{aligned}
& \text { Control } \\
& \text { I/R model } \\
& \text { Gal } 3 \mathrm{mg} / \mathrm{kg} \\
& \mathrm{CC} \\
& \mathrm{CC}+\mathrm{Gal} 3 \mathrm{mg} / \mathrm{kg}
\end{aligned}
$$

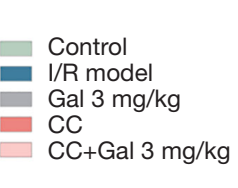

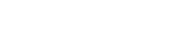

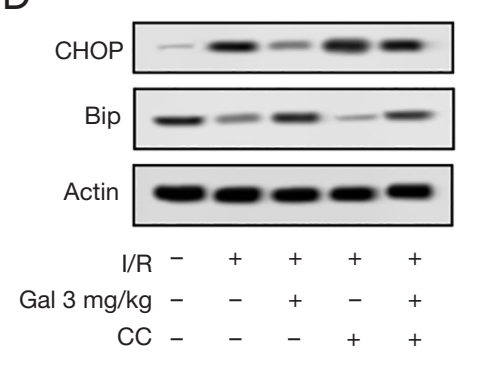

$\mathrm{CC}-\mathrm{C}_{+}+$

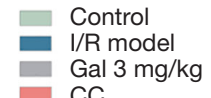

- $\mathrm{CC}$

CC+Gal $3 \mathrm{mg} / \mathrm{kg}$
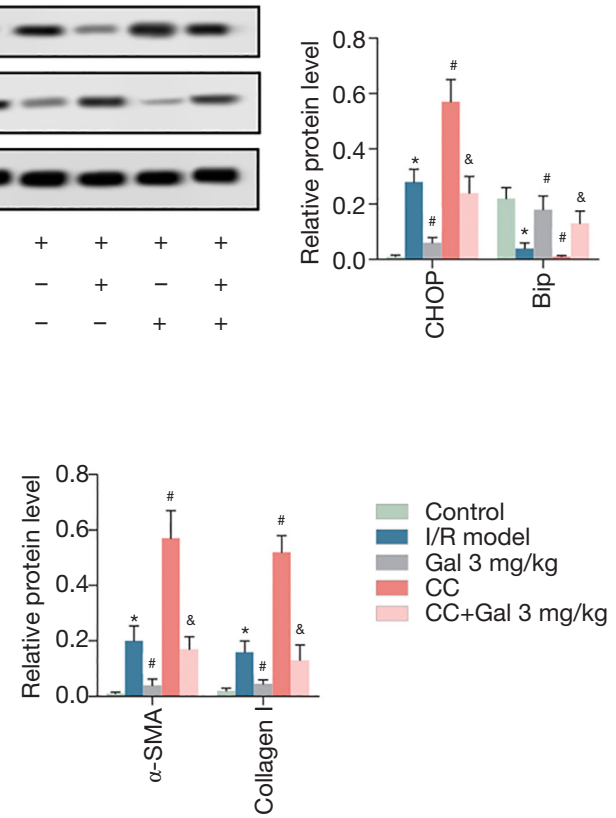

Figure $4 \mathrm{Gal}$ improves myocardial I/R injury by activating AMPK/Nrf2 pathway. (A) Rats were grouped into five groups (10 in each group): Sham group, I/R model, I/R + Gal (1 mg/kg), I/R + Gal (3 mg/kg), and I/R + Aspirin (20 mg/kg) group. The protein levels of AMPKa1, Nrf2, and HO-1 were measured by western blotting assay. Actin was used as an internal reference. ${ }^{*}, \mathrm{P}<0.05$ vs. sham group; ${ }^{*}, \mathrm{P}<0.05$ vs. I/R group. (B,C,D,E,F) Rats were grouped into five groups (10 in each group): Sham group, I/R model, I/R + Gal (3 mg/kg), I/R + CC $(200 \mu \mathrm{M})$, and $\mathrm{I} / \mathrm{R}+\mathrm{Gal}(3 \mathrm{mg} / \mathrm{kg})+\mathrm{CC}(200 \mu \mathrm{M})$ group. (B) The protein levels of AMPKa1, Nrf2, and HO-1 were measured by western blotting assay. (C) LVESV and FS. (D) The protein levels of CHOP and BiP were measured by western blotting assay. (E) The mRNA level of Caspase 3 was measured by RT-qPCR. (F) The protein levels of $\alpha$-SMA and collagen I were measured by western blotting assay. Actin was used as an internal reference. ${ }^{*}, \mathrm{P}<0.05$ vs. sham group; ${ }^{*}, \mathrm{P}<0.05$ vs. I/R group; ${ }^{\mathrm{k}}, \mathrm{P}<0.05$ vs. I/R + CC group. 
improvement on myocardial damage caused by I/R. Feng et al. found that schisandra can significantly improve cardiac function, reduce apoptosis, inhibit ERS, and attenuate MI/R-induced myocardial damage by activating AMPK signaling pathway (34). In addition to this, Zhang et al. found that ghrelin, a cardioprotective peptide, protects the heart from ischemia/reperfusion (I/R) injury by activating the AMPK signaling pathway and inhibiting endoplasmic reticulum stress (ERS)-induced damage and apoptosis (36). Similarly, Hou et al. found that hyperoside (Hyp) increased myocardial ATP levels, reduced myocardial oxidative damage, and reduced cardiomyocyte apoptosis by activating the Nrf2 signaling pathway (37). Moreover, Wang et al. found that Crocin attenuated I/R-induced ERS, left ventricular dysfunction and myocardial infarction in mice by inhibiting the miR-34a/Sirt1/Nrf2 pathway (35). Similar to the above results, this study found that I/R inhibited the expression of AMPK and Nrf2-related proteins (AMPK $\alpha 1$, $\mathrm{Nrf2}$ and HO-1). Interestingly, Gal treatment promoted the activation of the AMPK and Nrf2 pathways in a dosedependent manner, thereby improving I/R-induced cardiac dysfunction, endoplasmic reticulum stress-related apoptosis, and myocardial fibrosis.

Collectively, Gal improves I/R-induced cardiac dysfunction, ERS-related apoptosis, and myocardial fibrosis by suppressing AMPK/Nrf2 pathways. Overall, these findings support the use of $\mathrm{Gal}$ as a potential therapeutic drug for the treatment of I/R injury.

\section{Acknowledgments}

None.

\section{Footnote}

Conflicts of Interest: The authors have no conflicts of interest to declare.

Ethical Statement: The authors are accountable for all aspects of the work in ensuring that questions related to the accuracy or integrity of any part of the work are appropriately investigated and resolved. All animal experiments were performed in accordance with the NIH Guide for the Care and Use of Laboratory Animals and were approved by the Medical Ethics Committee of Sichuan Provincial People's Hospital.

\section{References}

1. Wang Q, Liu GP, Xue FS, et al. Combined Vagal Stimulation and Limb Remote Ischemic Perconditioning Enhances Cardioprotection via an Anti-inflammatory Pathway. Inflammation 2015;38:1748-60.

2. Cai ZP, Parajuli N, Zheng X, et al. Remote ischemic preconditioning confers late protection against myocardial ischemia-reperfusion injury in mice by upregulating interleukin-10. Basic Res Cardiol 2012;107:277.

3. Huang G, Hao F, Hu X. Downregulation of microRNA-155 stimulates sevoflurane-mediated cardioprotection against myocardial ischemia/reperfusion injury by binding to SIRT1 in mice. J Cell Biochem 2019;120:15494-505.

4. Liao YH, Xia N, Zhou SF, et al. Interleukin-17A contributes to myocardial ischemia/reperfusion injury by regulating cardiomyocyte apoptosis and neutrophil infiltration. J Am Coll Cardiol 2012;59:420-9.

5. Turer AT, Hill JA. Pathogenesis of myocardial ischemiareperfusion injury and rationale for therapy. Am J Cardiol 2010;106:360-8.

6. Kapur NK, Reyelt L, Swain L, et al. Mechanical Left Ventricular Unloading to Reduce Infarct Size During Acute Myocardial Infarction: Insight from Preclinical and Clinical Studies. J Cardiovasc Transl Res 2019;12:87-94.

7. Zhan G, Zhou J, Liu R, et al. Galanthamine, Plicamine, and Secoplicamine Alkaloids from Zephyranthes candida and Their Anti-acetylcholinesterase and Anti-inflammatory Activities. J Nat Prod 2016;79:760-6.

8. Dos Santos TC, Gomes TM, Pinto BAS, et al. Naturally Occurring Acetylcholinesterase Inhibitors and Their Potential Use for Alzheimer's Disease Therapy. Front Pharmacol 2018;9:1192.

9. Ray RS, Rai S, Katyal A. Cholinergic receptor blockade by scopolamine and mecamylamine exacerbates global cerebral ischemia induced memory dysfunction in C57BL/6J mice. Nitric Oxide 2014;43:62-73.

10. Mavropoulos SA, Khan NS, Levy ACJ, et al. Nicotinic acetylcholine receptor-mediated protection of the rat heart exposed to ischemia reperfusion. Molecular medicine (Cambridge, Mass) 2017;23:120-33.

11. Görlach A, Klappa P, Kietzmann T. The endoplasmic reticulum: folding, calcium homeostasis, signaling, and redox control. Antioxid Redox Signal 2006;8:1391-418.

12. Lee WJ, Chien MH, Chow JM, et al. Nonautophagic cytoplasmic vacuolation death induction in human PC- 
$3 \mathrm{M}$ prostate cancer by curcumin through reactive oxygen species -mediated endoplasmic reticulum stress. Sci Rep 2015;5:10420.

13. Cullinan SB, Diehl JA. Coordination of ER and oxidative stress signaling: the PERK/Nrf2 signaling pathway. Int J Biochem Cell Biol 2006;38:317-32.

14. Cullinan SB, Zhang D, Hannink M, et al. Nrf2 is a direct PERK substrate and effector of PERK-dependent cell survival. Mol Cell Biol 2003;23:7198-209.

15. Chen XJ, Ren SM, Dong JZ, et al. Ginkgo biloba extract-761 protects myocardium by regulating Akt/Nrf2 signal pathway. Drug design, development and therapy 2019;13:647-55.

16. Tian L, Cao W, Yue R, et al. Pretreatment with Tilianin improves mitochondrial energy metabolism and oxidative stress in rats with myocardial ischemia/reperfusion injury via AMPK/SIRT1/PGC-1 alpha signaling pathway. J Pharmacol Sci 2019;139:352-60.

17. Ahmed LA. Pharmacological preconditioning with nicorandil and pioglitazone attenuates myocardial ischemia/reperfusion injury in rats. European Journal of Pharmacology 2011;663:51-8.

18. Jin JK, Blackwood EA, Azizi K, et al. ATF6 Decreases Myocardial Ischemia/Reperfusion Damage and Links ER Stress and Oxidative Stress Signaling Pathways in the Heart. Circ Res 2017;120:862-75.

19. Lesnefsky EJ, Chen Q, Tandler B, et al. Mitochondrial Dysfunction and Myocardial Ischemia-Reperfusion: Implications for Novel Therapies. Annu Rev Pharmacol Toxicol 2017;57:535-65.

20. Attisano L, Wrana JL. Signal transduction by the TGFbeta superfamily. Science 2002;296:1646-7.

21. Zhang WH, Fu SB, Lu FH, et al. Involvement of calciumsensing receptor in ischemia/reperfusion-induced apoptosis in rat cardiomyocytes. Biochem Biophys Res Commun 2006;347:872-81.

22. Lei Y, Wang S, Ren B, et al. CHOP favors endoplasmic reticulum stress-induced apoptosis in hepatocellular carcinoma cells via inhibition of autophagy. PLoS One 2017;12:e0183680.

23. Ibrahim SM, Al-Shorbagy MY, Abdallah DM, et al. Activation of alpha7 Nicotinic Acetylcholine Receptor Ameliorates Zymosan-Induced Acute Kidney Injury in BALB/c Mice. Sci Rep 2018;8:16814.

24. Ma SL, Tang NLS, Wat KHY, et al. Effect of CYP2D6 and CYP3A4 Genotypes on the Efficacy of Cholinesterase Inhibitors in Southern Chinese Patients
With Alzheimer's Disease. Am J Alzheimers Dis Other Demen 2019;34:302-7.

25. Njoku I, Radabaugh HL, Nicholas MA, et al. Chronic treatment with galantamine rescues reversal learning in an attentional set-shifting test after experimental brain trauma. Exp Neurol 2019;315:32-41.

26. Wang T, Zhu H, Hou Y, et al. Galantamine reversed early postoperative cognitive deficit via alleviating inflammation and enhancing synaptic transmission in mouse hippocampus. Eur J Pharmacol 2019;846:63-72.

27. Liu X, Xu K, Yan M, et al. Protective effects of galantamine against Abeta-induced PC12 cell apoptosis by preventing mitochondrial dysfunction and endoplasmic reticulum stress. Neurochem Int 2010;57:588-99.

28. Miyazaki Y, Kaikita K, Endo M, et al. C/EBP homologous protein deficiency attenuates myocardial reperfusion injury by inhibiting myocardial apoptosis and inflammation. Arterioscler Thromb Vasc Biol 2011;31:1124-32.

29. Guo C, Zhang J, Zhang P, et al. Ginkgolide B ameliorates myocardial ischemia reperfusion injury in rats via inhibiting endoplasmic reticulum stress. Drug Des Devel Ther 2019;13:767-74.

30. Du XJ, Wei J, Tian D, et al. NEAT1 promotes myocardial ischemia-reperfusion injury via activating the MAPK signaling pathway. 2 J Cell Physiol 2019;234:18773-80.

31. Deng T, Wang Y, Wang C, et al. FABP4 silencing ameliorates hypoxia reoxygenation injury through the attenuation of endoplasmic reticulum stress-mediated apoptosis by activating PI3K/Akt pathway. Life Sci 2019;224:149-56.

32. Cui Y, Wang Y, Liu G. Protective effect of Barbaloin in a rat model of myocardial ischemia reperfusion injury through the regulation of the CNPY2PERK pathway. Int J Mol Med 2019;43:2015-23.

33. Shang L, Dong P, Du L, et al. SERP1 prevents hypoxiareoxygenation-induced $\mathrm{H} 9 \mathrm{c} 2$ apoptosis through activating JAK2/STAT3 pathway-dependent attenuation of endoplasmic reticulum stress. Biochem Biophys Res Commun 2019;508:256-62.

34. Feng Y, Lu Y, Liu D, et al. Apigenin-7-O-beta-d-(-6"'p-coumaroyl)-glucopyranoside pretreatment attenuates myocardial ischemia/reperfusion injury via activating AMPK signaling. Life Sci 2018;203:246-54.

35. Wang X, Yuan B, Cheng B, et al. Crocin Alleviates Myocardial Ischemia/Reperfusion-Induced Endoplasmic Reticulum Stress via Regulation of miR-34a/Sirt1/Nrf2 Pathway. Shock 2019;51:123-30. 
36. Zhang GG, Cai HQ, Li YH, et al. Ghrelin protects heart against ERS-induced injury and apoptosis by activating AMP-activated protein kinase. Peptides 2013;48:156-65.

37. Hou JY, Liu Y, Liu L, et al. Protective effect of hyperoside

Cite this article as: Hou X, Fu M, Cheng B, Kang Y, Xie D. Galanthamine improves myocardial ischemia-reperfusioninduced cardiac dysfunction, endoplasmic reticulum stressrelated apoptosis, and myocardial fibrosis by suppressing AMPK/Nrf2 pathway in rats. Ann Transl Med 2019;7(22):634. doi: $10.21037 /$ atm.2019.10.108 on cardiac ischemia reperfusion injury through inhibition of ER stress and activation of Nrf2 signaling. Asian Pac J Trop Med 2016;9:76-80. 